\title{
Selección de estrategias para la implementación de Mejoras de Procesos Software
}

\author{
Brenda Liliana Durón del Villar \\ Centro de Investigación en Matemáticas \\ brenda.duron@cimat.mx
}

\section{Mirna Ariadna Muñoz Mata \\ Centro de Investigación en Matemáticas mirna.munoz@cimat.mx}

Resumen: La mejora de procesos software se ha convertido en la forma más lógica y obvia de dirigir la creciente necesidad de aumentar la competitividad en las empresas de desarrollo de software. Desafortunadamente no todas las implementaciones de mejoras en los procesos tienen el rendimiento deseado, debido a que los modelos y estándares existentes centran su atención en qué actividades implementar sin abordar el cómo implementarlas. Sin embargo, la identificación de qué actividades implementar no es suficiente y el conocimiento del cómo implementarlas es requerido para el éxito de la implementación de iniciativas de Mejoras de Procesos Software (MPS) en las empresas de desarrollo de software. En este trabajo se presenta los resultados tanto de una revisión del estado actual en la implementación de MPS lograda mediante una revisión literaria así como los resultados de un análisis de empresas locales de Zacatecas logrado mediante la aplicación de entrevistas. Finalmente, se presenta una propuesta para el establecimiento de estrategias para la implementación de MPS basadas en los aspectos contextuales en los cual el software es desarrollado y entregado, tal que, pueda ser seleccionada una estrategia acorde a las necesidades y cultura de trabajo de la organización. 
Palabras clave: Mejoras de procesos software, Estrategias de Implementación, empresas desarrollo de software.

\title{
Selection of strategies for the implementation of Software Process Improvement
}

\begin{abstract}
Software process improvement has become the most logical and obvious way to address the growing need of increasing the competitiveness in software development companies. Unfortunately not all process improvements implementations have the desired results, because existing models and standards focus their attention on what activities implemented without addressing how to implement them. However, identifying what activities to implement is not enough; the knowledge of how to implement them is required for a successful implementation of Software Process Improvement (SPI) initiatives in organizations. This paper presents the results of reviewing both the current state of the implementation of SPI using a literature review and by performing interviews in software development organizations of Zacatecas. Finally, based on the findings of this analysis, the paper includes a proposal to develop strategies for the implementation of SPI based on the contextual aspects in which the software is developed and delivered, so that the strategy according to the organization needs and work culture can be selected.
\end{abstract}

Keywords: Software Process Improvement, Implementation strategies, software development organizations.

\section{Introducción}

Para una empresa de desarrollo de software es de vital importancia crear estrategias para ser competitiva frente a sus compositores. Es por esto que, las organizaciones implementan iniciativas de Mejoras de Procesos Software para incrementar la calidad del producto reduciendo el tiempo de entrega y los costos de producción (Cugola \& Ghezzi, 1998) con lo cual consiguen esa ventaja competitiva. Como consecuencia de esto, la mejora de procesos de software se ha convertido en un mecanismo importante ya que impulsa la competitividad y eficiencia de las organizaciones de software (Cuevas et al., 2002).

En este contexto, en los últimos años se han desarrollado modelos y 
estándares para la apoyar a las organizaciones en la implementación de mejoras de los procesos de desarrollo de software, como el modelo CMMI, o la norma ISO/IEC15504, estos modelos y estándares centran su atención en qué actividades implementar sin abordar el cómo implementar estas actividades. No obstante, el conocimiento de cómo implementarlos es requerido para una exitosa implementación de estas iniciativas de Mejora de Procesos Software (MPS) en las empresas. Por lo que, un problema actual con la mejora de procesos de software no es la falta de estándares o modelos, si no la falta de estrategias efectivas de implementación de esos estándares o modelos (Niazi, Wilson \& Zowghi, 2005).

Es en este contexto y con el objetivo de encontrar el cómo realizar una implementación exitosa de MPS se realiza esta investigación donde se pretende establecer y proponer un conjunto de estrategias tal que las organizaciones puedan elegir la que mejor les sirva teniendo en cuenta su contexto organizacional. Este trabajo se organiza de la siguiente manera, la sección 2 presenta el estado actual de MPS; la sección 3 muestra una investigación de MPS realizada en el ámbito local; la sección 4 presenta la propuesta de estrategias para una implementación de MPS y finalmente en la sección 5 se muestran algunas conclusiones y trabajo futuro.

\section{Estado Actual de Mejora de Procesos Software}

Para conocer el estado actual de MPS se realizó una Revisión Sistemática (RS) en 5 de las principales fuentes electrónicas de artículos científicos. Una revisión sistemática de la literatura permite identificar, evaluar, interpretar y sintetizar todas las investigaciones existentes y relevantes en un tema de interés particular (Feiler \& Humphrey, 1992); por esto los resultados de la 
revisión sistemática se tomaron como punto de partida para esta investigación. En la Figura 1, se muestra el procedimiento para selección de estudios.

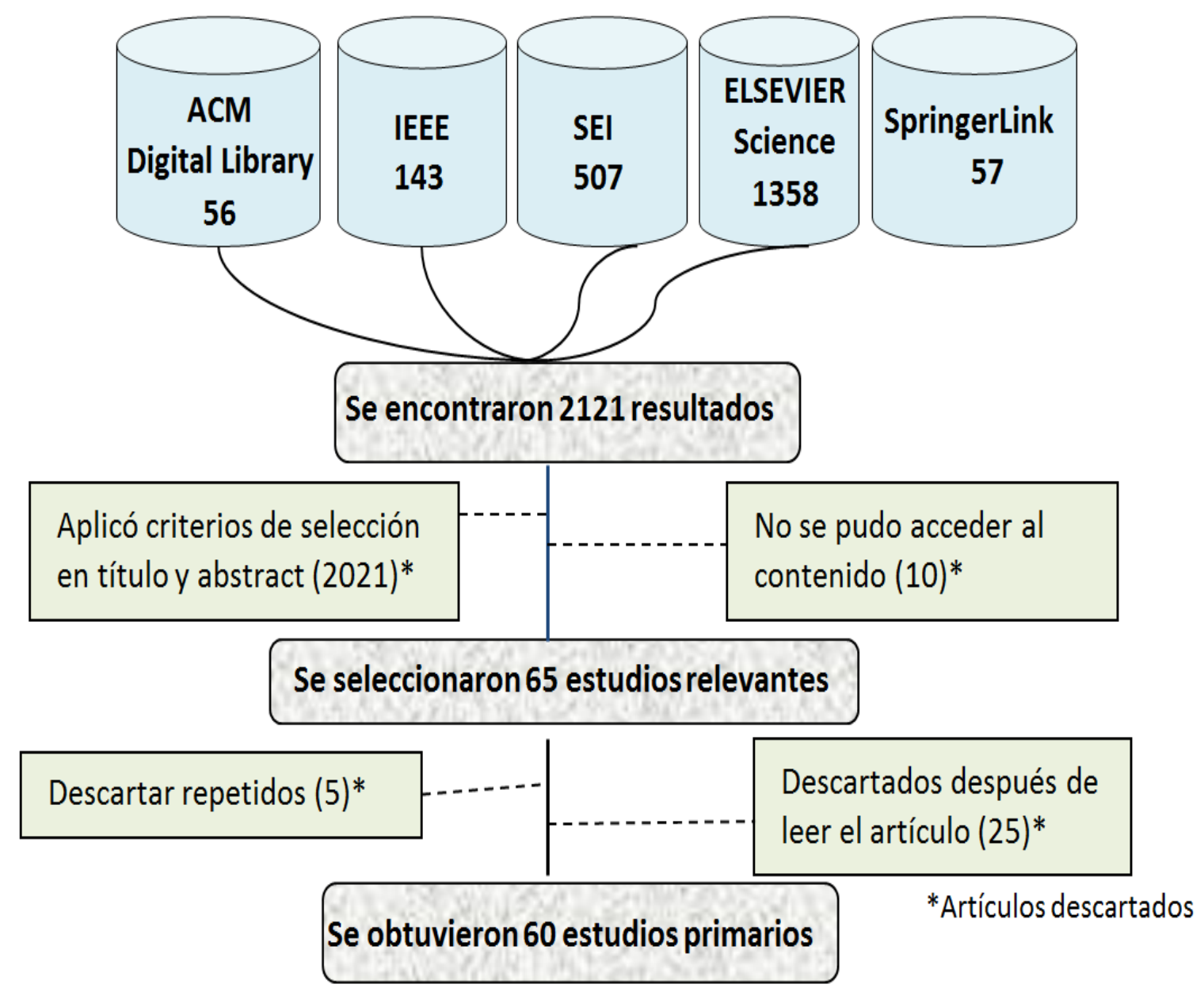

figura 1. Selección de estudios de la Revisión Sistemática

\subsection{Análisis de los Resultados}

Estudios realizados muestran que el $67 \%$ de los gestores solicitan orientación sobre cómo implementar las iniciativas de mejoras de procesos software, en lugar de qué actividades implementar (Herbsleb \& Goldenson, 1996). Lo que 
ha causado el aumento de la investigación en materia de implementación de mejoras de procesos en los últimos años.

Dentro del análisis de resultados se identificaron los siguientes problemas comunes en las estrategias de implementación de MPS: no se tienen claros los objetivos de la MPS; no se presta suficiente atención a los factores organizacionales que promueven o inhiben las mejoras de procesos y no se toma en cuenta el capital humano, y por lo tanto, está propenso a un inesperado 0 indeseado rendimiento y/o comportamiento. Se encontraron además que varios autores destacan listas de barreras y desmotivadores en las actividades de MPS (Niazi, Babar \& Verner, 2010; Baddoo \& Hall, 2003), siendo las más recurrentes las que se muestran en la Tabla 1.

Existen también varios estudios de campo así como investigaciones donde se busca encontrar los factores críticos para una exitosa implementación de MPS esto con la finalidad de garantizar que las iniciativas de implementación sean exitosas (Dyba Tore 2003; Montoni \& Rocha 2010). Dentro de los factores identificados se encuentran la orientación de negocios, líderes y gerencias comprometidos e involucrados en el proceso, participación activa de los empleados, uso de medidas y capacitación y conciencia de la organización. Finalmente se identificaron y analizaron los métodos/modelos/frameworks existentes y se analizaron sus características. 
Entendimiento del proceso de mejoras

* Falta de recursos dedicados a la implementación de mejoras

* Estructura organizacional y política

Restricciones de tiempo y calendario

* Falta de gestión del proyecto de mejoras

- Experiencias negativas previas

* Falta de una metodología definida para la Falta de evidencia de los beneficios implementación de mejoras

* Falta de comunicación

* Iniciativas de mejoras impuestas

* Falta de soporte, herramientas y formación

tabla 1. Barreras y desmotivadores en la implementación de Mejora de Procesos Software encontrados en la Revisión Sistemática

\section{Investigación en Empresas Locales}

\subsection{Resultados}

Como parte de este trabajo de investigación se realizaron entrevistas con empresas locales desarrolladoras de software de Zacatecas con la finalidad de contrastar con resultados reales los resultados obtenidos en la revisión sistemática. Para obtener la información de las empresas locales se elaboró un cuestionario como guía en las entrevistas para poder obtener la las experiencias en MPS de las empresas.

A continuación se listan los resultados de la investigación de las empresas locales enfocando en características, hallazgos, necesidades, mejores prácticas:

a) Características 
Como resultado se han entrevistado 8 empresas de Zacatecas. En la Figura 2 se muestran las características de ellas basado en servicios que ofrecen, empleados por empresa y experiencia en MPS.

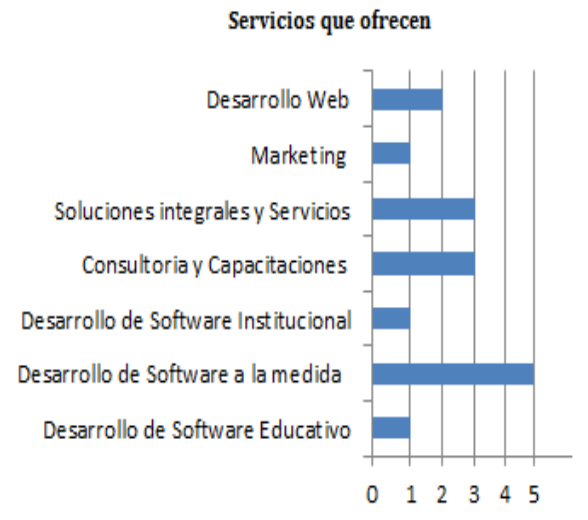

- Empresas que ofrecen ese servicio
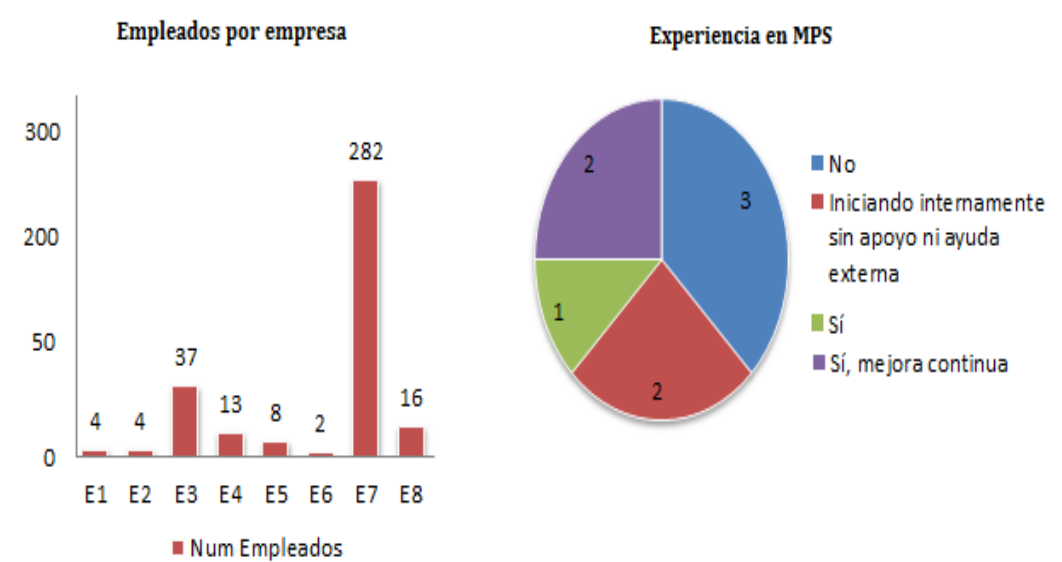

figura 2. Caracterización de empresas encuestadas

Como se observa en la Figura 2: Como parte de los resultados además se identificaron hallazgos, necesidades y mejores prácticas de las empresas como se lista a continuación.

b) Hallazgos

- Solamente 3 de las empresas desarrolladoras de software analizadas cuentan con experiencia en MPS.

- Dos empresas están en un ciclo de mejora continua.

- Las dos empresas restantes apenas están reconociendo esta necesidad de mejorar.

- La empresa restante no considera necesario en su ámbito la adopción de algún modelo o método ya que consideran que no se adecuan a su forma de trabajo.

- Por otra parte ,cabe resaltar que existe un conocimiento pobre de un enfoque de procesos, por lo que no se cree que sea necesario implementar modelos y estándares aunado a que las empresas no cuentan con 
presupuesto suficiente para actividades de mejora lo que impide que estas empresas puedan obtener los beneficios de la mejora de procesos.

c) Necesidades

Cabe resaltar, por un lado, que las empresas entrevistadas con poca experiencia o nula en MPS piden dos cosas principales: 1) presupuesto para poder asignar personal a la mejora de procesos y 2) tiempo para implementar proyectos de mejora.

d) Mejores prácticas

Por otro lado, como resultado de las entrevistas a empresas que ya han tenido experiencia en MPS se obtuvieron algunas prácticas que les han ayudado al éxito en su implementación de las que destacan:

- Establecer inicialmente los objetivos, definiendo por qué se quiere una implantación de mejora procesos.

- Convencer a la alta gerencia para que sean promotores, patrocinadores y guías de la implementación de mejoras.

- Visualizar el beneficio que se obtendrá con la mejora de procesos.

- Apostar por el capital intelectual de la empresa.

\subsection{Contraste de resultados revisión literaria e investigación en empresas locales}

En este apartado se muestran las Barreras y desmotivadores en la implementación de Mejora de Procesos Software encontrados en la Revisión Sistemática y que coincidieron en las entrevistas del ámbito local, estas barreras y desmotivadores se encuentran en la Tabla 2. 
Barreras

Entendimiento del proceso de mejoras

* Falta de una metodología definida para la implementación de mejoras

* Falta de soporte, herramientas y formación Falta de evidencia de los beneficios

tabla 2. Coincidencia entre Barreras y desmotivadores para la implementación de MPS.

\section{Propuesta de Estrategias para la Implementación de MPS}

Derivado de las experiencias obtenidas en la revisión de literatura como en el estudio de pymes del ámbito local de Zacatecas, se identificaron diferentes estrategias que han guiado y servido en la implementación de MPS y que pueden ayudar en la parte del cómo realizar las actividades de mejora. Sin embargo, aun cuando se han reportado los beneficios de éstas, esto no garantiza que sean adecuadas para todas las empresas, es decir, no existe una regla única que nos asegure el éxito (Jeners, Clarke, O'Connor, Buglione \& Lepmets,

2013).

De acuerdo con (Petersen \& Wohlin, 2009), el proceso de desarrollo de software idealmente debería estar en armonía con el contexto en el cual el software es desarrollado y entregado. Esto significa que con el fin de establecer si una solución específica puede tener éxito, es necesario describir el contexto tan completa y precisamente como sea posible para el caso de estudio. Este trabajo se enfoca en la elección de las estrategias correctas para 
implementar mejoras de procesos adecuadas a cada contexto específico como a continuación se describe:

Se inicia con prefase en la que se confirma, mediante un checklist, que la organización tienen identificado como trabajan, cuáles son los objetivos de negocio que busca alcanzar; cuál es su rendimiento y su objetivo al implementar la mejora de procesos. Posteriormente, la empresa realiza una comparación de su entorno con los entornos establecidos en la propuesta por medio de categorías y así se les pueda proporcionar aquella estrategia que se adecue a sus necesidades. Para el establecimiento del conjunto de estrategias se toman como base los seis aspectos contextuales propuestos (Petersen \& Wohlin 2009), considerados como aspectos a tener en cuenta para una implementación de mejora de procesos en cualquier organización: producto, proceso, prácticas y técnicas, recurso humano, organización y mercado, estos aspectos se describen brevemente en la Tabla 3.

\begin{tabular}{llllll}
\hline \multicolumn{1}{c}{ Producto } & Proceso & $\begin{array}{c}\text { Prácticas, } \\
\text { Herramientas, } \\
\text { Técnicas }\end{array}$ & $\begin{array}{c}\text { Recurso } \\
\text { humano }\end{array}$ & Organización & Mercado \\
\hline Madurez & Actividades & Herramientas & Roles & Modelodela & Número de \\
Calidad & Flujode & Prácticas y & Experiencia & organización & clientes \\
Tamaño & trabajo & técnicas & & Unidadesde & Segmento del \\
Tipode sistema & Artefactos & & negocio & mercado \\
Personalización & & & Certificaciones & Estrategias \\
Lenguajede & & & Distribución & Restricciones \\
programación & & & & &
\end{tabular}

tabla 3. Aspectos contextuales en los cual el software es desarrollado y entregado. 
Además, para lograr el establecimiento de este conjunto de estrategias, se analizaran los principales métodos/modelos de implementación de estrategias; las lecciones aprendidas y las barreras y desmotivadores identificados tanto en la revisión literaria como en el estudio de las pymes de la región. El procedimiento propuesto para la selección de la estrategia se muestra en la Figura 3.

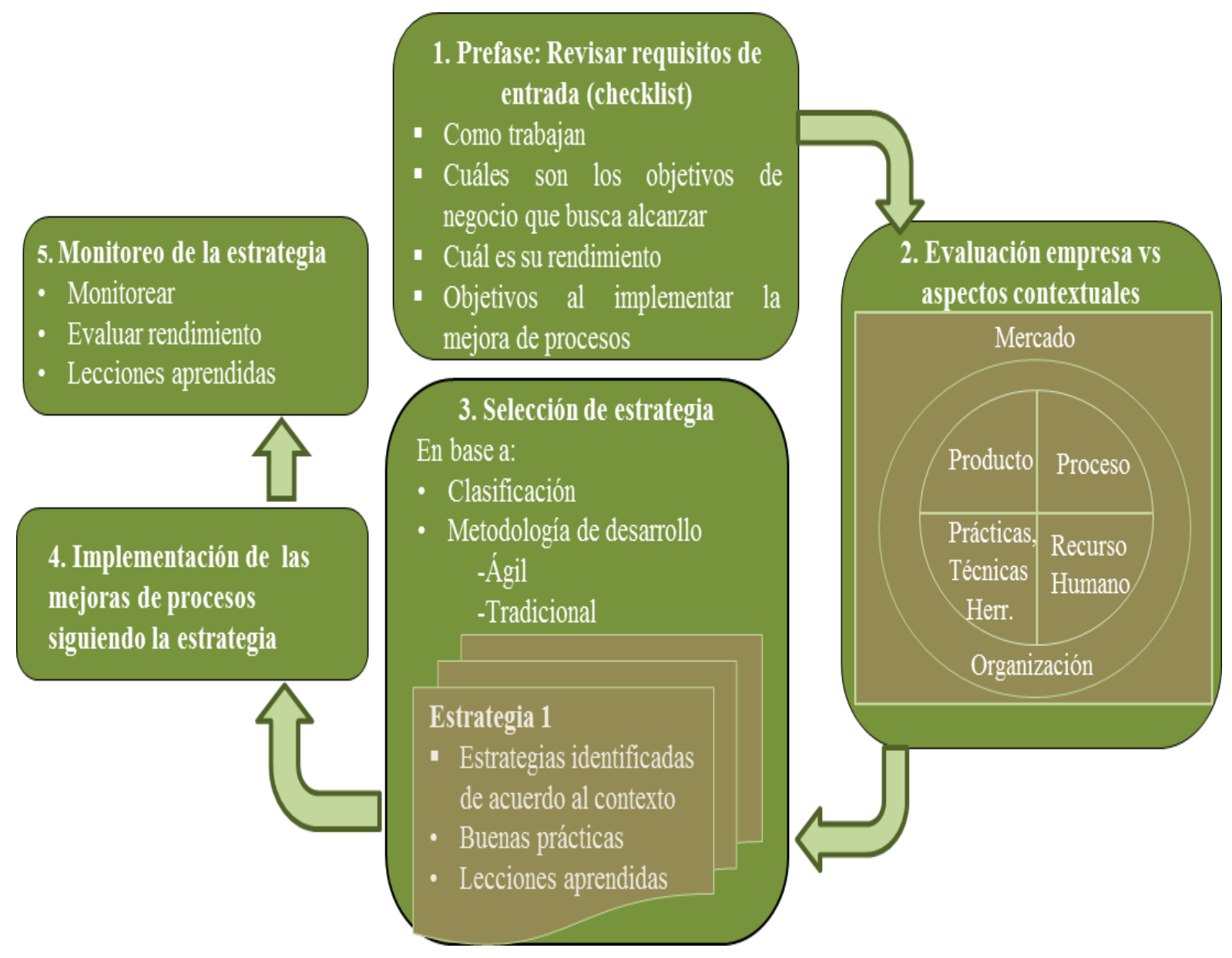

figura 3. Propuesta de selección de estrategias para MPS. 


\section{Conclusiones y Trabajo Futuro}

Las empresas han asimilado que la calidad del producto de software está directamente relacionada con la calidad del proceso utilizado para su desarrollo; y es por esto que, el interés en este campo de la ingeniería de software está en constante aumento reflejado en el desarrollo de nuevas técnicas, herramientas, metodologías y métodos que apoyan a las organizaciones en el desarrollo de productos de calidad e implementación de mejoras en los procesos. Sin embargo, aún con la existencia de modelos, métodos y frameworks ya desarrollados para la implementación de mejoras y una serie de documentos en los que se identifican barreras, factores críticos para el éxito, así como motivadores y desmotivadores en la mejora de procesos software; sigue presentándose una falta de conocimiento en estrategias específicas enfocadas en cómo vencer todas esas barreras, como tratar los factores críticos.

Por lo tanto, se ha detectado además un nicho muy importante en la identificación de aquellas mejores prácticas de los modelos, métodos y estándares existentes que puedan marcar el camino que apoya en la conducción de una implementación de mejoras exitosa. Es importante resaltar que el éxito de MPS requiere de tolerancia al fracaso y que este fracaso es un prerrequisito esencial para el aprendizaje. Por lo tanto, las empresas deben ser capaces de convertir los fallos y problemas inesperados en oportunidades de aprendizaje.

Como trabajo futuro se vinculará cada aspecto contextual con categorías que permitan una comparación entre las empresas de una forma más sencilla. También se realizará el análisis de los modelos de implementación de MPS existentes identificados en la literatura y junto con las estrategias y lecciones aprendidas para crear el conjunto de estrategias de la propuesta. Una vez terminada la propuesta se probará su validez en empresas de desarrollo de software. 


\section{Referencias}

Baddoo Nathan, Hall Tracy (2003). De-motivators for software process improvement: an analysis of practitioners' views, Journal of Systems and Software, Volume 66, Issue 1, Pages 23-33

Calvo-Manzano Jose A., Cuevas Gonzalo, Gómez Gerzón, Mejia Jezreel, Muñoz Mirna, San Feliu Tomás, (2009) Methodology for process improvement through basic components and focusing on the resistance to change, Journal of Software Maintainance and evolution: Research and practice.

Cuevas G., De Amescua A., San Feliu T., Arcilla M., Cerrada J.A., CalvoManzano J.A., García M., (2002), Gestión del Proceso Software, Universitaria Ramon Areces, pp. 472.

Cugola G. and Ghezzi C., (1998),Software Processes: A Retrospective and a Path to the Future, Software Process: Improvement and Practice, vol. 4, no. 3, pp. 101-12

Dyba Tore (2003). Factors of software process improvement success in small and large organizations: an empirical study in the scandinavian context, Proceedings of the 9th European software engineering conference, Pages 148 $-157$

Feiler, P., Humphrey, W., (1992) Software process development and enactment:Concepts and definitions. SEl, Carnegie Mellon University, CMU/SEl92-TR-004. Pittsburgh, Pennsylvania, USA

Herbsleb, J. D. and Goldenson, D. R. (1996). A systematic survey of CMM experience and results. 18th international conference on software engineering (ICSE-18). Germany.

Jeners, S., Clarke, P., O'Connor, R. V., Buglione, L., and Lepmets, M. Harmonizing SoftwareDevelopment Processes with Software Development Settings - A Systematic Approach, 20th European Conference on Systems, Software and Services Process Improvement (EuroSPI 2013), CCIS Vol. 364

Kai Petersen, Claes Wohlin ,(2009) Context in Industrial Software Engineering Research, Third International Symposiumm on Empirical Software Engineering and Measurement

Montoni M.A., Rocha A.R. (2010). Applying Grounded Theory to Understand Software Process Improvement Implementation, Seventh International Conference on the Quality of Information and Communications Technology, Pages 25 - 34 
Niazi Mahmood, Wilson David, Zowghi Didar (2005). A maturity model for the implementation of software process improvement: an empirical study, Journal of Systems and Software, Volume 74, Issue 2, Pages 155-172.

Niazi Mahmood, Babar Muhammad Ali, Verner June M. (2010). Software Process Improvement barriers: A cross-cultural comparison, Journal of Information and Software Technology, Volume 52, Issue 11, Pages 1204-1216 


\section{Notas biográficas:}

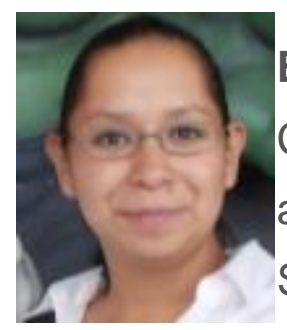

Brenda Liliana Durón del Villar recibió el grado de ingeniero en Computación de la Universidad Autónoma de Zacatecas; actualmente está estudiando la maestría en Ingeniería de Software en el Centro de Investigación en Matemáticas CIMAT. Su interés es en la implementación exitosa de mejoras de procesos software así como las estrategias que ayuden a minimizar la resistencia al cambio en las empresas de desarrollo de software.

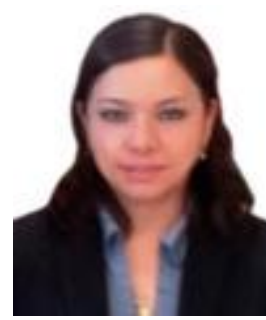

Mirna Ariadna Muñoz Mata, Doctor en Informática por la Universidad Politécnica de Madrid, en Madrid España, con mención de "Doctorado Europeo". Ha realizado una estancia posdoctoral en la Universidad Carlos III de Madrid, España. Actualmente es investigador del Centro de Investigación en Matemáticas (CIMAT) - Unidad Zacatecas en el área de Ingeniería de Software y es miembro del grupo de investigación Cátedra de Mejora de Procesos Software en el Espacio Iberoamericano (MPSEI), donde participa en proyectos internacionales de investigación con entidades educativas y de gobierno y de vinculación con la industria. Ha participado en proyectos con la empresa everis consulting. Ha participado en el equipo de traducción oficial al español reconocida por el SEI del libro CMMI-DEV v1.2 y 1.3. Es miembro del comité científico de diversos congresos internacionales como: CISTI (2009-2012), del coloquio de investigación multidisciplinaria del Instituto Tecnológico de Orizaba (2011), del Infonor Chile 2012, del CERMA (2012) y de la revista internacional RISTI (2010-2012). Ha publicado diversos artículos técnicos en temas relacionados con la gestión de proyectos, implementación de mejora de procesos software, entornos multi-modelo y modelos y estándares de calidad. 
Es autora del libro Metodología Multimodelo para Implementar Mejoras de Procesos Software. Sus áreas de interés son: implementación de mejora de procesos software enfocando en la reducción de la resistencia al cambio, entornos multi-modelo, gestión del conocimiento y gestión del cambio en la mejora de procesos software, gestión de proyectos software, trabajo en equipo y modelos y estándares de calidad.

\section{(c) (i) (2) (2)}

Esta obra está bajo una licencia de Creative Commons Reconocimiento-NoComercial-Compartirlgual 2.5 México. 